\title{
Association of biobehavioral factors with non-coding RNAs in cervical cancer
}

\author{
Qiyu Liu ${ }^{1, \S}$, Chong Lu ${ }^{1, \S}$, Wanjun Dai ${ }^{1, \S}, \mathrm{Ke} \mathrm{Li}^{1}$, Jing $\mathrm{Xu}^{1}$, Yunke Huang ${ }^{1}$, Guiling $\mathbf{L i}^{1,2,3}$, \\ Yu Kang ${ }^{1,2,3, *}$, Anil K. Sood ${ }^{4, *}$, Congjian $\mathrm{Xu}^{1,2,3, *}$ \\ ${ }^{1}$ Department of Obstetrics and Gynecology of Shanghai Medical School, Fudan University, Shanghai, China; \\ ${ }^{2}$ Department of Integrated Traditional Chinese and Western Medicine, Obstetrics and Gynecology Hospital, Fudan University, \\ Shanghai, China; \\ ${ }^{3}$ Shanghai Key Laboratory of Female Reproductive Endocrine Related Diseases, Shanghai, China; \\ ${ }^{4}$ Departments of Gynecologic Oncology, Cancer Biology, and Center for RNA Interference and Noncoding RNA, University of \\ Texas, M.D. Anderson Cancer Center, TX, United States.
}

\begin{abstract}
Summary In order to elucidate the mechanisms underlying the biobehavioral factors responsible for cervical cancer from the perspective of IncRNAs. Tumor samples were obtained from patients with stage Ib-IIb squamous cervical cancer, which were divided into high- and lowrisk groups according to biobehavioral risk factors. A IncRNA + mRNA microarray was performed, and the results were validated using qRT-PCR. Gene ontology (GO), pathway, and IncRNA-mRNA co-expression analysis were performed to predict the potential functions of the differentially expressed transcripts. 1,621 IncRNAs and 1,345 mRNAs were found to be differentially expressed between the high-risk and low-risk groups. The results of the qRTPCR validation were in $100 \%$ agreement with the microarray analysis results. GO analysis revealed that the transcripts showing significantly different expression were mainly associated with various aspects of immune response. Pathway analysis indicated that systemic lupus erythematosus signaling was the most significantly down-regulated pathway in the highrisk group. Co-expression analysis indicated NONHSAT002712, NONHSAT095060, and TCONS_00026535 had significant correlations with ZNF683 and BTLA, which were found to be associated with the GO term "adaptive immune response". The levels of genome-wide IncRNAs are significantly altered in cervical tumors from patients with higher biobehavioral risk factors.
\end{abstract}

Keywords: Cervical cancer, biobehavioral factor, psychoimmunology, adaptive immune response

\section{Introduction}

Cervical cancer is the second most commonly diagnosed cancer and the third leading cause of cancer

\footnotetext{
${ }^{\S}$ These authors contributed equally to this work.

*Address correspondence to:

Drs. Yu Kang and Congjian Xu, Department of Obstetrics and Gynecology of Shanghai Medical School, Fudan University, 413 Zhaozhou Road, Shanghai 200011, China.

E-mail: yukang@fudan.edu.cn (Kang Y), xucongjian@fudan. edu.cn $(\mathrm{Xu} \mathrm{CJ})$

Dr. Sood AK, Departments of Gynecologic Oncology, Cancer Biology, and Center for RNA Interference and Noncoding RNA, University of Texas, M.D. Anderson Cancer Center, 1515 Holcombe Blvd, Houston, TX 77030, United States.

E-mail: asood@mdanderson.org
}

death among females in developing countries. Among the females diagnosed with cervical cancer, many are diagnosed at the advanced stages of the disease where they have limited treatment options and show poor prognosis (1). There were an estimated 98,900 new cervical cancer cases and 30,500 related deaths in China in 2015 (2).

Cervical cancer patients experience significantly more depression and anxiety than the general population (3). Women who receive results of an abnormal Pap smear or a positive HPV DNA test may often experience unwarranted fear, distress, and anxiety about cervical cancer (4). A recent analysis indicates that $25.7 \%$ of early stage cervical cancer (ECC) cases and $22.2 \%$ of locally advanced cervical cancer (LACC) cases have elevated anxiety levels (5). 
In addition, cervical cancer survivors are more likely to have a lower quality of life (QOL) and higher levels of depression and anxiety (26\% and $28 \%$, respectively) compared to other cancer survivors (6).

Biobehavioral factors such as depression, anxiety, and stressful life events have long been suspected to underlie cancer progression (7). Animal studies have also revealed that behavioral stress can promote the progression of ovarian cancer, breast cancer, pancreatic cancer, and several other types of malignant tumors $(8,9)$. The mechanisms underlying the effects of biobehavioral stress on cancer progression have been studied through psychoneuroimmunology $(7,10)$, and these studies have shown that biobehavioral factors influence the neuroendocrine regulation of hormones (i.e., catecholamine neurotransmitters, dopamine, adrenaline, and noradrenaline), which may impair the immune response and contribute to cancer onset and development.

Long noncoding RNAs (lncRNAs) are transcripts longer than 200 nucleotides with no apparent proteincoding role. LncRNAs are involved in numerous important biological processes such as chromatin modification, genomic imprinting, and enzymatic activity regulation (11). The overexpression, deficiency, or mutation of IncRNAs has been implicated in various malignant tumors and other human diseases (12). However, few studies have evaluated the changes and potential functions of IncRNAs in cervical cancer patients experiencing higher psychological stress.

In the current study, we performed a highthroughput analysis to compare the lncRNA and mRNA expression profiles between high- and lowpsychological stress cervical cancer groups. Our aim was to discover the mechanisms underlying the potential psychoimmunological effects of behavioral stress on cervical cancer from the perspective of lncRNAs. Our findings provide new insights into the psychological epidemiology of cervical cancer.

\section{Materials and Methods}

\subsection{Patients}

Women older than 18 years of age with an abnormal Pap test result suspected for cervical carcinoma were determined to be potentially eligible for this study. The study inclusion was confirmed after the histologic diagnosis of squamous cell carcinoma of the uterine cervix. Patients with a previous cancer diagnosis, regular use of a systemic steroidal medication in the last 4 months, comorbidities known to alter the immune response (such as autoimmune diseases), or the inability to accurately answer questions were excluded. This study was approved by the Ethics Committee of the Obstetrics and Gynecology Hospital affiliated to Fudan University.

\subsection{Psychological measures}

\subsubsection{Depression}

Depressive symptoms were assessed using the Center for Epidemiologic Studies Depression Scale (CESD) and the Zung's Self-rating Depression Scale (SDS). The CESD is a validated 20 -item scale that assesses depressive symptoms occurring in the prior week. Scores of 16 or higher indicate a high biobehavioral risk (13). Four subscales of CESD have been confirmed by factor analysis: depressed affect, vegetative depression, positive affect, and interpersonal relationships. On the other hand, the SDS is a 20 -item self-reported scale that assesses psychological and somatic symptoms of depression. Cut-off scores for the SDS were as follows: $<50=$ normal, 50 to $59=$ mild depression, 60 to $69=$ moderate depression, and $>69$ $=$ severe depression $(14)$.

\subsubsection{Social support}

Social support was assessed by the Chinese version of the Social Support Rating Scale (SSRS), which demonstrates good validity and reliability among Chinese populations (15). The SSRS consists of ten items that measure three dimensions of social support: objective support, subjective support, and support utilization.

\subsubsection{Anxiety}

The Zung's Self-rating Anxiety Scale (SAS) was used to quantify the level of anxiety (16). The self-reported questionnaire contained 20 items. Cut-off scores were as follows: $<45=$ normal, 45 to $59=$ mild to moderate anxiety, 60 to $74=$ marked to severe anxiety, and $>75=$ extreme anxiety.

\subsubsection{Sleep quality}

The Pittsburgh Sleep Quality Index (PSQI) is a 19-item self-reported questionnaire used to assess the type and frequency of sleep disturbances occurring in the prior month (17). A global score greater than 5 indicated poor sleep.

\subsection{Tissue collection and RNA extraction}

Six cervical carcinoma samples were harvested during the laparoscopic surgery from each study participant. All the samples were rapidly frozen in liquid nitrogen, followed by storage at $-80^{\circ} \mathrm{C}$. Total RNA was extracted from the frozen tumor tissues using the mirVana RNA Isolation Kit (Ambion). RNA concentration and purity were then determined using the NanoDrop ND2000 Spectrophotometer (Thermo Fisher Scientific). RNA integrity was determined by standard denaturing 
agarose gel electrophoresis.

\subsection{Microarray analysis}

Total RNA was labeled using the Quick-Amp Labeling Kit, One-Color (Agilent Technologies) and hybridized onto the Agilent Human lncRNA Array (4*180K). The chip detected 46506 human lncRNAs and 30656 human mRNAs. Hybridization signals were detected using the Microarray Scanner (Agilent p/n G2505C). Agilent Feature Extraction Software was utilized to extract the raw data. Quantile normalization and subsequent processing of the data were carried out using GeneSpring Software version 12.0 (Agilent Technologies). Differentially expressed genes were defined as those with an absolute value of fold change $(\mathrm{FC})>2$ and a $p$-value $<0.05$ (Student's $t$-test). Microarray profiling was performed by OE Biotech, Shanghai, China.

\subsection{Quantitative real-time PCR assay}

Total RNA was reverse transcribed into cDNA using the PrimerScript RT Kit with gDNA Eraser (Takara, Shiga, Japan) according to the manufacturer's standard protocols. Quantitative real-time PCR (qRT-PCR) was performed on an Applied Biosystems Vii ${ }^{\mathrm{TM}}$ A7 System (Life Technologies, Tokyo, Japan). Each $20-\mu \mathrm{L}$ reaction contained $10 \mu \mathrm{L}$ of SYBR Premix Ex Taq II $(2 \times)$ (Takara), $2 \mu \mathrm{L}$ of cDNA, $0.8 \mu \mathrm{L}$ of the forward primer, $0.8 \mu \mathrm{L}$ of the reverse primer, and $6 \mu \mathrm{L}$ of $\mathrm{dH}_{2} \mathrm{O}$. The PCR cycling conditions were as follows: incubation at $95^{\circ} \mathrm{C}$ for $10 \mathrm{~min}$, followed by 40 cycles at $95^{\circ} \mathrm{C}$ for 10 $\mathrm{s}$ and $60^{\circ} \mathrm{C}$ for $30 \mathrm{~s}$. Each sample was run in triplicate for analysis. Melting curve analysis was performed to validate the specificity of each PCR product. The expression levels of the mRNAs and lncRNAs were normalized to the GAPDH level and calculated using the $2^{-\Delta \Delta \mathrm{Ct}}$ method.

\subsection{Gene ontology and pathway analysis}

Gene Ontology (GO) analysis and Kyoto Encyclopedia of Genes and Genomes (KEGG) pathway analysis were used to determine the potential roles of the differentially expressed mRNAs. GO analysis (http://geneontology. org/) provides three-structured networks of defined terms that describe genes and their properties, which includes information on their biological processes, cellular components, and molecular functions. We also adopted the KEGG pathway (http://www.genome.jp/kegg/) to predict the biological functions of the target genes.

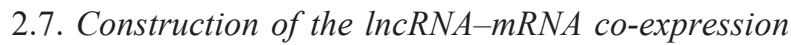 network}

The lncRNA-mRNA co-expression network was constructed to explore the relationship between the
IncRNAs and mRNAs. For each pair of genes, the Pearson correlation coefficient (PCC) was calculated, and the pairs with significant correlations ( $\mathrm{PCC}>$ 0.90) were chosen to construct the network. Cytoscape Software version 3.4.0 (U.S. National Institute of General Medical Sciences) was used to illustrate the coexpression network.

\section{Results}

\subsection{Patient characteristics}

Six squamous cervical carcinoma patients who had undergone primary surgical resection between November 2014 and September 2015 were enrolled in this study. The clinical characteristics of the patients are shown in Supplementary Table S1 (http://www.biosciencetrends. com/action/getSupplementalData.php?ID=18). All the tumor samples were confirmed to be stage Ib1-IIb squamous cervical carcinomas.

Psychological factors were measured during the presurgical clinic visits 1 to 7 days prior to tumor resection. Based on the established threshold of CESD $\geq 16$, three participants were determined to show high levels of psychological risk factors. The only measured property that differed significantly between the two groups was the level of depressive scores (CESD, $p=0.0327$; SDS, $p=0.0399$ ).

\subsection{LncRNA and $m R N A$ expression profiles}

Using a 2 or 0.5 -fold change as the cut-off, a total of 1,621 lncRNAs were found to be differentially expressed between the high-risk and low-risk groups; of these, 510 were up-regulated and 1,111 were downregulated in the high-risk group (Supplementary Table S2, http://www.biosciencetrends.com/action/ getSupplementalData.php?ID=19). Table 1 shows the top ten up-regulated and down-regulated lncRNAs. Using the same criteria, we found 1,345 mRNAs showing differential expression between the high-risk and low-risk groups; of these, 325 were up-regulated and 1,020 were down-regulated in the high-risk group (Supplementary Table S3, http://www.biosciencetrends. com/action/getSupplementalData.php? ID=20). The top ten up-regulated and down-regulated mRNAs are listed in Table 2. The volcano plot of the differentially expressed lncRNAs and mRNAs is shown in Supplementary Figure S1 (http://www.biosciencetrends. com/action/getSupplementalData.php?ID=21). Hierarchical clustering analysis was performed to categorize the lncRNAs and mRNAs based on their expression levels in the microarray (Figure 1).

\subsection{Quantitative real-time PCR validation}

To validate the microarray results by real-time PCR 
Table 1. Top ten up-regulated and down-regulated IncRNAs in the high-risk group

\begin{tabular}{|c|c|c|c|c|}
\hline LncRNAs & Source database & Fold change (up-regulated) & Fold change (down-regulated) & $P$-value \\
\hline NONHSAT026375 & NONCODE v4 & 83.38818 & & 0.004714203 \\
\hline NONHSAG051449 & NONCODE v4 & 56.622337 & & 0.007290442 \\
\hline NONHSAT006501 & NONCODE v4 & 54.330788 & & 0.028635195 \\
\hline NR_040072.1 & RefSeq & 29.504402 & & 0.00305648 \\
\hline NR_045603.1 & RefSeq & 21.908543 & & 0.034243103 \\
\hline NONHSAT029137 & NONCODE v4 & 16.355997 & & 0.001712117 \\
\hline NONHSAT142855 & NONCODE v4 & 15.255895 & & 0.00395211 \\
\hline NONHSAT006296 & NONCODE v4 & 13.86696 & & 0.044070113 \\
\hline NONHSAT026373 & NONCODE v4 & 13.66836 & & 0.049752276 \\
\hline NONHSAT029135 & NONCODE v4 & 12.5354595 & & 0.00199061 \\
\hline NONHSAT097055 & NONCODE v4 & & 21.751446 & 0.008822498 \\
\hline NONHSAT059677 & NONCODE v4 & & 20.691536 & 0.025407365 \\
\hline NONHSAT130141 & NONCODE v4 & & 20.616901 & 0.00182173 \\
\hline TCONS_12_00010069 & broadlincRNA & & 19.297148 & 0.001462107 \\
\hline TCONS_12_00010131 & broadlincRNA & & 18.281479 & 0.038280915 \\
\hline NONHSAT-145020 & NONCODE v4 & & 15.2914095 & 0.004498216 \\
\hline NONHSAT029287 & NONCODE v4 & & 15.215009 & 0.014417922 \\
\hline NONHSAT040550 & NONCODE v4 & & 14.950378 & 0.014418174 \\
\hline NONHSAT026886 & NONCODE v4 & & 14.94323 & 0.002577477 \\
\hline NONHSAG023913 & NONCODE v4 & & 14.099488 & 0.005995478 \\
\hline
\end{tabular}

Table 2. Top ten up-regulated and down-regulated mRNAs in the high-risk group

\begin{tabular}{|c|c|c|c|}
\hline LncRNAs & Fold change (up-regulated) & Fold change (down-regulated) & $P$-value \\
\hline SPRR1A & 68.54885 & & 0.037800513 \\
\hline A2ML1 & 47.889496 & & 0.01010738 \\
\hline VTCN1 & 47.843727 & & 0.012803975 \\
\hline PSCA & 38.76277 & & 0.001021038 \\
\hline SH3BGRL2 & 25.990252 & & 0.01292145 \\
\hline MLPH & 20.98717 & & $1.38 \mathrm{E}-04$ \\
\hline POF1B & 18.330408 & & 0.03179252 \\
\hline RHCG & 17.668486 & & 0.025924051 \\
\hline DNAH14 & 15.095662 & & 0.011291295 \\
\hline FAM189A2 & 12.6118145 & & 0.041048728 \\
\hline SLC44A5 & & 63.488415 & $9.77 \mathrm{E}-04$ \\
\hline CXCL13 & & 22.780071 & 0.004740034 \\
\hline GLDC & & 22.185339 & 0.002045446 \\
\hline CXCL11 & & 18.150942 & 0.019471126 \\
\hline ELAVL2 & & 14.732332 & 0.020086708 \\
\hline IFNG & & 13.957739 & 0.007875251 \\
\hline KLRC3 & & 12.96415 & 0.001003543 \\
\hline MMP7 & & 12.581658 & 0.027674025 \\
\hline UBD & & 12.484317 & 0.04345015 \\
\hline IL12RB2 & & 12.357619 & 0.010752848 \\
\hline
\end{tabular}

quantification, we selected three mRNAs and two lncRNAs from the six cervical cancer samples that were subjected to the microarray analysis. The qRTPCR results showed that the expression of lncRNA NONHSAT097055 and the CCL5, CXCL9, and HIST1H2AM mRNAs was significantly decreased $(p$-values for all <0.05) in the high-risk samples compared to that in the low-risk samples. By contrast, the expression of IncRNA NONHSAT029137 was significantly increased in the high-risk group ( $p<$ 0.05) (Figure 2). The qRT-PCR results were consistent with those obtained from the microarray analysis, thus confirming the microarray results.

\subsection{Gene enrichment and pathway analysis}

GO analysis was performed for all the differentially expressed mRNAs to identify the potential functions of their coding transcripts. We found that the mRNAs showing significant differential expression between the high-risk and low-risk groups were mainly associated with the immune response (GO:0006955), adaptive immune response (GO:0002250), regulation of immune response (GO:0050776), protein heterodimerization activity (GO:0046982), and transmembrane signaling receptor activity (GO:0004888), which are all involved in various biological processes and molecular functions. 
A

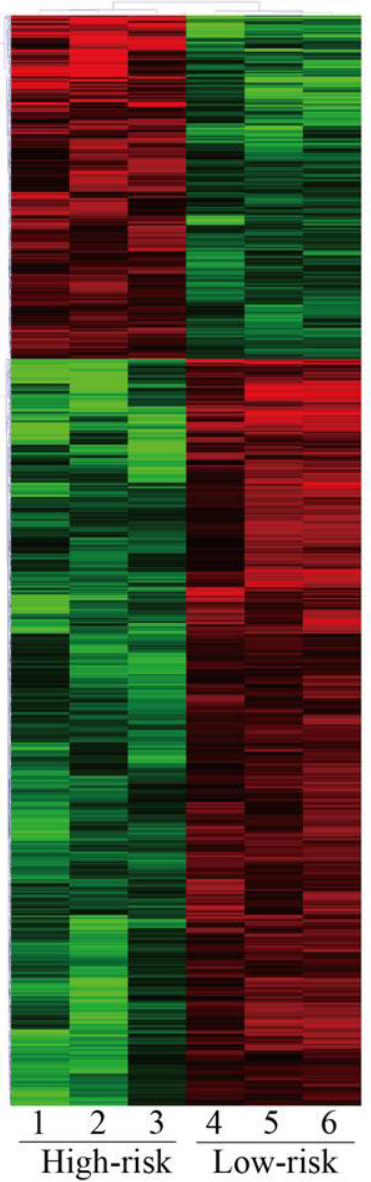

B

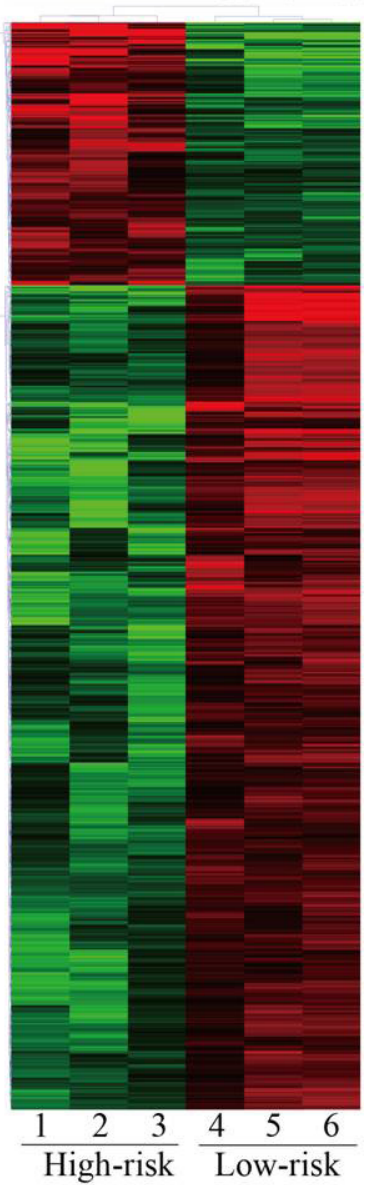

Figure 1. Heat map and hierarchical clustering of the differentially expressed IncRNAs (A) and mRNAs (B) in the high-risk and low-risk groups. The red and green bars indicate the expression levels above and below the relative expression across all the samples.

The detailed results are presented in Figures 3 A-C.

KEGG pathway analysis indicated that the most significantly enriched pathways consisted of those that regulate systemic lupus erythematosus (hsa05322), antigen processing and presentation (hsa04612), and natural killer (NK) cell mediated cytotoxicity (hsa04650). The top 20 enriched pathways of the differentially expressed mRNAs are shown in Figure 3 D.

\subsection{Construction of the IncRNA-mRNA co-expression network}

In order to investigate the correlation between the differentially expressed lncRNAs and mRNAs, the IncRNA-mRNA co-expression network was constructed. In total, 509 lncRNAs and 230 mRNAs were included in the co-expression network (Supplementary Table S4, http://www.biosciencetrends. com/action/getSupplementalData.php?ID=22). To draw the co-expression network, we selected several mRNAs that were found to be involved in the dysregulated functions and pathways in the GO and

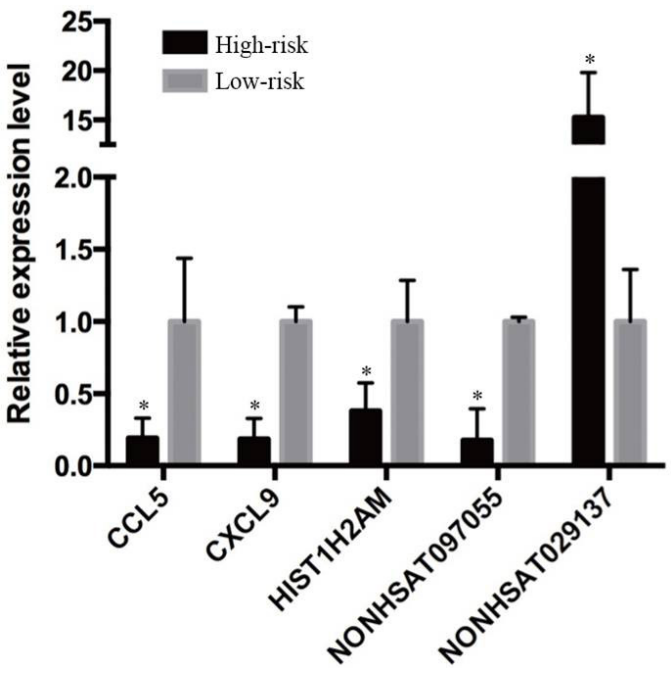

Figure 2. Validation of microarray data using quantitative real-time PCR. Three mRNAs (CCL5, CXCL9, and HIST1H2AM) and two lncRNAs (NONHSAT097055 and NONHSAT029137) were selected and analyzed by qRT-PCR to validate their expression levels. The relative expression level of the target mRNA/lncRNA was normalized, and data displayed in the histograms are expressed as means \pm standard deviation (SD), ${ }^{*} p<0.05$ upon comparison between the highrisk and low-risk groups.

KEGG pathway analysis. As shown in Figure 4, 52 IncRNAs were found to interact with two mRNAs (CBL and PRKCQ) in the KEGG term of "T cell receptor signaling pathway" (A), 51 lncRNAs were found to interact with two mRNAs (CXCR5 and IL12B) in the KEGG term of "cytokine-cytokine receptor pathway" (B), and 39 lncRNAs were found to interact with two mRNAs (BTLA and ZNF683) in the GO term of "adaptive immune response" (C).

\section{Discussion}

In this study, we profiled the expression of lncRNAs and mRNAs in cervical cancer samples from patients with different psychological risk levels by microarray analysis. We found that 1,621 lncRNAs and 1,345 mRNAs were differentially expressed between the two groups. GO and KEGG pathway analysis was performed to predict the potential functions of the differentially expressed mRNAs. Moreover, we predicted the target genes of the differentially expressed lncRNAs by constructing lncRNA-mRNA coexpression networks. Based on our results, we predicted that some of the differentially expressed genes play important roles in the psychological stress-induced psychoimmunological effects on cervical cancer.

There are a substantial number of studies on the effects of the chronic activation of the stress response on the immune response associating with cancer initiation and progression (10). Psychological stress has been shown to suppress the non-specific and specific components of the immune response, such as antigen presentation, NK cell activity, $\mathrm{T}$ cell 
A

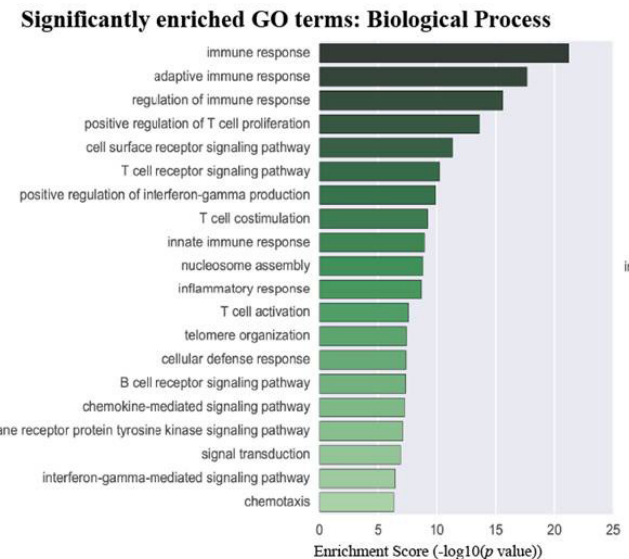

C

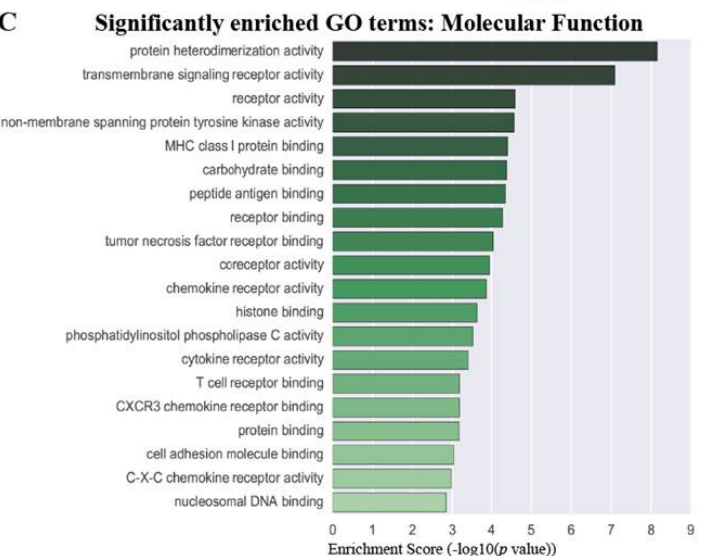

B Significantly enriched GO terms: Cellular Component

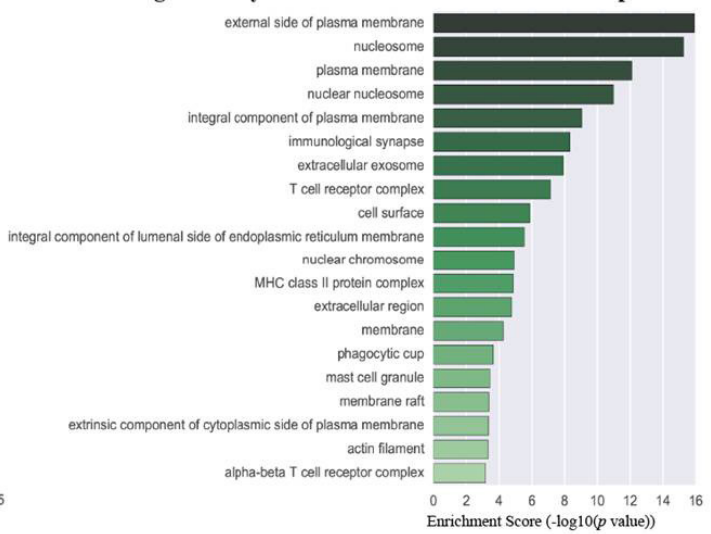

D

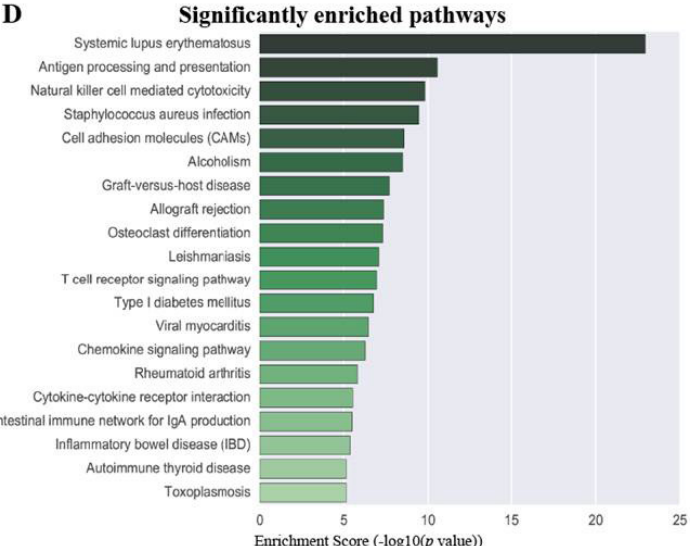

Figure 3. Functional prediction of the differentially expressed mRNAs based on GO enrichment analysis (A-C) and KEGG pathway analysis (D). GO analysis covered three domains: biological processes, cellular components, and molecular functions.

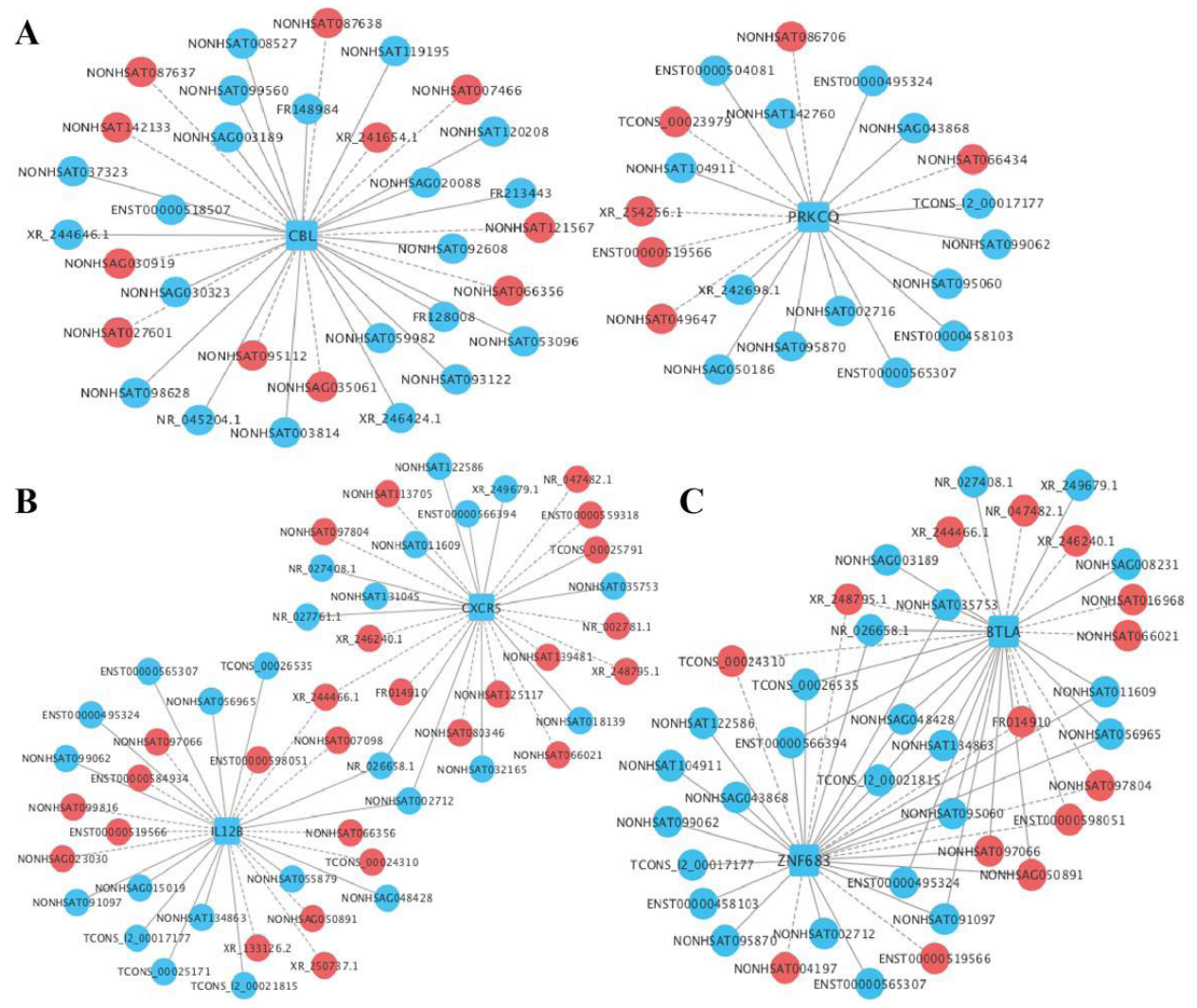

Figure 4. LncRNA-mRNA co-expression network. (A) 52 lncRNAs were found to interact with two mRNAs in the meaningful "T cell receptor signaling pathway". (B) 51 lncRNAs were found to interact with two mRNAs in the meaningful "cytokine-cytokine receptor pathway". (C) 39 lncRNAs were found to interact with two mRNAs in the GO term of "adaptive immune response". Square nodes represent the mRNAs, and round nodes represent the lncRNAs. The up-regulated and down-regulated genes are indicated in red and blue, respectively. Solid lines indicate a positive correlation, and dashed lines indicate a negative correlation. 
proliferation, cytotoxic $\mathrm{T}$ cell activity, and production of inflammatory cytokines via mechanisms that involve adrenergic and glucocorticoid-mediated pathways (7).

Cancers caused by DNA tumor viruses might be more affected by psychological and immunological factors than those induced by chemical carcinogens (10). Researchers have identified persistent infection with a high-risk human papillomavirus (HPV) as the cause of over $99 \%$ of cervical cancers (18). Fang et al. also reported that higher levels of perceived stress are associated with impaired HPV-specific immune response in women with cervical dysplasia (19). Moreover, a recent clinical study showed that bereavement is associated with an increased risk of HPV infection and cervical cancer in Sweden (20). Stressful life events might also be associated with impaired immune surveillance and possibly poor control over HPV infection and thus increase the risk of cervical cancer.

The ZNF683 (Zinc finger protein 683) and BTLA (B and T Lymphocyte Associated) mRNAs were found to be associated with the GO term "adaptive immune response". ZNF683, also known as Hobit (Homolog of Blimp-1 in T cells), is a transcription factor that mediates a transcriptional program in tissue-resident memory $\mathrm{T}$ (Trm) and natural killer $\mathrm{T}$ (NKT) cells $(21,22)$. Hobit mediates the development and retention of Trm cells and NKT cells in non-lymphoid organs and other nonbarrier tissues and may provide immediate immunological protection against re-infections. BTLA is an immunoglobulin-like molecule belonging to the B7 family, which relays inhibitory signals that suppress the immune response (23). Moreover, the interaction between BTLA and its ligand has been reported to be actively involved in the adaptive immune response (24). In our study, we constructed a lncRNA-mRNA co-expression network to predict the potential functions of the differentially expressed lncRNAs. Our results showed that several lncRNAs, such as NONHSAT002712, NONHSAT095060, and TCONS_00026535, had significant correlations with ZNF683 and BTLA, suggesting that these lncRNAs had roles in the regulation of the adaptive immune response.

The major limitation of this study is that the data were obtained from a small sample size. In the future, larger studies will be required to establish the generality of these findings. Nevertheless, this study is novel in that it identified genome-wide lncRNAs correlating with psychological stress specifically in cervical cancer tissues and thus provides new insights into the psychological epidemiology of cervical cancer.

\section{Acknowledgements}

This work was supported by the National Key Research and Development Program of China (2016YFC1303100, 2016YFC1303102), the National Natural Science Foundation of China (81472423),
Shanghai Municipal Health and Family Planning Commission (ZYKC201701020), and the Natural Science Foundation of Shanghai (14430723000).

\section{References}

1. Torre LA, Bray F, Siegel RL, Ferlay J, Lortet-Tieulent J, Jemal A. Lortet-Tieulent J, Jemal A. Global cancer statistics, 2012. CA Cancer J Clin. 2015; 65:87-108.

2. Chen W, Zheng R, Baade PD, Zhang S, Zeng H, Bray F, Jemal A, Yu XQ, He J. Cancer statistics in China, 2015. CA Cancer J Clin. 2016; 66:115-132.

3. Bradley S, Rose S, Lutgendorf S, Costanzo E, Anderson B. Quality of life and mental health in cervical and endometrial cancer survivors. Gynecol Oncol. 2006; 100:479-486.

4. Herzog TJ, Wright JD. The impact of cervical cancer on quality of life--the components and means for management. Gynecol Oncol. 2007; 107:572-577.

5. Mantegna G, Petrillo M, Fuoco G, Venditti L, Terzano S, Anchora LP, Scambia G, Ferrandina G. Long-term prospective longitudinal evaluation of emotional distress and quality of life in cervical cancer patients who remained disease-free 2-years from diagnosis. BMC Cancer. 2013;13:127.

6. Osann K, Hsieh S, Nelson EL, Monk BJ, Chase D, Cella D, Wenzel L. Factors associated with poor quality of life among cervical cancer survivors: implications for clinical care and clinical trials. Gynecol Oncol. 2014;135: 266272.

7. Antoni MH, Lutgendorf SK, Cole SW, Dhabhar FS, Sephton SE, McDonald PG, Stefanek M, Sood AK. The influence of bio-behavioural factors on tumour biology: Pathways and mechanisms. Nat Rev Cancer. 2006; 6:240248.

8. Thaker PH, Han LY, Kamat AA, et al. Chronic stress promotes tumor growth and angiogenesis in a mouse model of ovarian carcinoma. Nat Med. 2006; 12:939-944.

9. Cole SW, Nagaraja AS, Lutgendorf SK, Green PA, Sood AK. Sympathetic nervous system regulation of the tumour microenvironment. Nat Rev Cancer. 2015; 15:563-572.

10. Reiche EM, Nunes SO, Morimoto HK. Stress, depression, the immune system, and cancer. Lancet Oncol. 2004; 5:617-625.

11. Quinn JJ, Chang HY. Unique features of long non-coding RNA biogenesis and function. Nat Rev Genet. 2016; 17:47-62.

12. Esteller M. Non-coding RNAs in human disease. Nat Rev Genet. 2011; 12:861-874.

13. Ensel W. Measuring Depression: The CES-D Scale. In: Lin N, Dean A,Ensel W (eds): Social Support, Life Events, and Depression. New York:Academic Press, 1986; 51-70.

14. Zung WW, Magruder-Habib K, Velez R, Alling W. The comorbidity of anxiety and depression in general medical patients: a longitudinal study. J Clin Psychiatry. 1990; 51(Suppl):77-80; discussion 81.

15. Dai W, Chen L, Tan H, Wang J, Lai Z, Kaminga AC, Li Y, Liu A. Association between social support and recovery from post-traumatic stress disorder after flood: A 1314 year follow-up study in uunan, China. BMC Public Health. 2016; 16:194.

16. Zung WW. A rating instrument for anxiety disorders. 
Psychosomatics. 1971; 12:371-379.

17. Buysse DJ, Reynolds CF, Monk TH, Berman SR, Kupfer DJ. The pittsburgh sleep quality index: A new instrument for psychiatric practice and research. Psychiatry Res. 1989; 28:193-213.

18. Moody CA, Laimins LA. Human papillomavirus oncoproteins: Pathways to transformation. Nat Rev Cancer. 2010;10:550-560

19. Fang CY, Miller SM, Bovbjerg DH, Bergman C, Edelson MI, Rosenblum NG, Bove BA, Godwin AK, Campbell DE, Douglas SD. Perceived stress is associated with impaired T-cell response to HPV16 in women with cervical dysplasia. Ann Behav Med. 2008; 35:87-96.

20. Lu D, Sundström K, Sparén P, Fall K, Sjölander A, Dillner J, Helm NY, Adami HO, Valdimarsdóttir U, Fang F. Bereavement is associated with an increased risk of HPV infection and cervical cancer: An epidemiological study in Sweden. Cancer Res. 2016; 76:643-651.

21. Mackay LK, Minnich M, Kragten NA, et al. Hobit and Blimp1 instruct a universal transcriptional program of tissue residency in lymphocytes. Science. 2016; 352: 459463.

22. Van Gisbergen KP, Kragten NA, Hertoghs KM, Wensveen FM, Jonjic S, Hamann J, Nolte MA, Van Lier RA. Mouse Hobit is a homolog of the transcriptional repressor Blimp-1 that regulates NKT cell effector differentiation. Nat Immunol. 2012; 13:864-871.

23. Yang C, Chen Y, Guo G, Li H, Cao D, Xu H, Guo S, Fei L, Yan W, Ning Q, Zheng L, Wu Y. Expression of $\mathrm{B}$ and $\mathrm{T}$ lymphocyte attenuator (BTLA) in macrophages contributes to the fulminant hepatitis caused by murine hepatitis virus strain-3. Gut. 2013; 62:1204-1213.

24. Derré L, Rivals JP, Jandus C, Pastor S, Rimoldi D, Romero P, Michielin O, Olive D, Speiser DE. BTLA mediates inhibition of human tumor-specific $\mathrm{CD} 8^{+} \mathrm{T}$ cells that can be partially reversed by vaccination. J Clin Invest. 2010; 120:157-167.

(Received December 29, 2017; Revised February 11, 2018; Accepted February 12, 2018) 